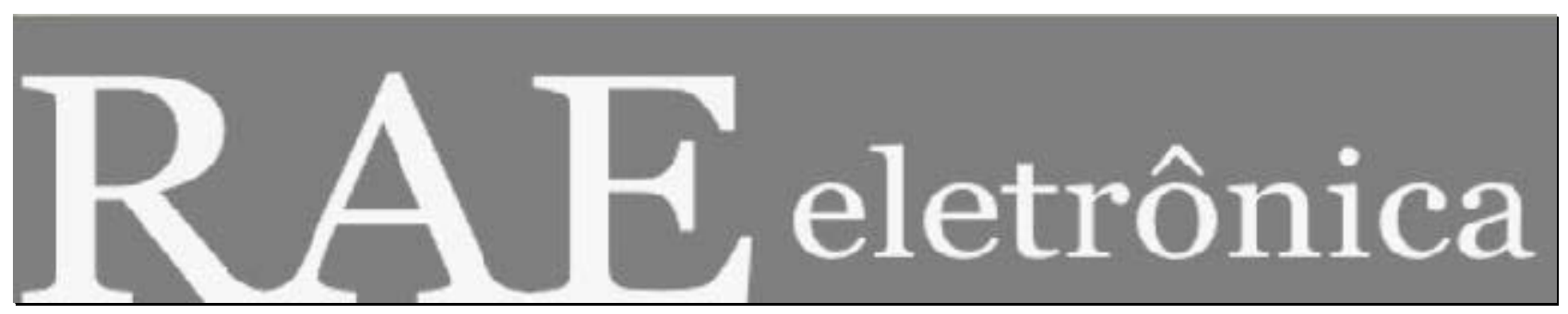

FÓRUM - SOCIOLOGIA ECONÔMICA

FORO - SOCIOLOGÍA ECONÓMICA

FORUM - ECONOMIC SOCIOLOGY

\title{
A PERSPECTIVA dos CUSTOS de TRANSAÇÃO NA FORMAÇÃO DE REDES DE COOPERAÇÃO
}
LA PERSPECTIVA DE LOS COSTOS DE TRANSACCIÓN EN LA FORMACIÓN DE REDES DE COOPERACIÓN

THE TRANSACTION-COSTS PERSPECTIVE IN THE FORMATION OF COOPERATIVE NETWORKS

Por:

ALSONES BALESTRIN, UNISINOS

ALESSANDRO PORPORATTI ARBAGE, UFSM

RAE-eletrônica, v. 6, n. 1, Art. 7, jan./jun. 2007

http://www.rae.com.br/eletronica/index.cfm?FuseAction=Artigo\&ID=3995\&Secao=FÓRUM\&Volume $=6 \&$ numero $=1 \& A n o=2007$

CCopyright, 2007, RAE-eletrônica. Todos os direitos, inclusive de tradução, são reservados. É permitido citar parte de artigos sem autorização prévia desde que seja identificada a fonte. A reprodução btal de artigos é proibida. Os artigos só devem ser usados para uso pessoal e nãocomercial. Em caso de dúvidas, consulte a redação: raeredacao@fgvsp.br.

A RAE-eletrônica é a revista on-line da FGV-EAESP, totalmente aberta e criada com o objetivo de agilizar a veiculação de trabalhos inéditos. Lançada em janeiro de 2002, com perfil acadêmico, é dedicada a professores, pesquisadores e estudantes. Para mais informações consulte o site www.rae.com.br/eletronica.

RAE-eletrônica

ISSN 1676-5648

(C)2007 Fundação Getulio Vargas - Escola de Administração de Empresas de São Paulo.

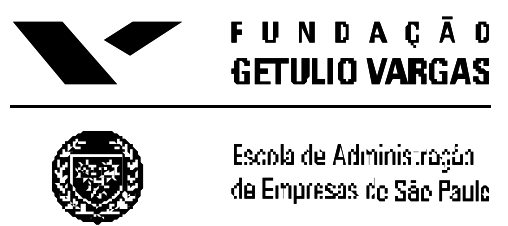


FÓRUM - SOCIOLOGIA ECONÔMICA

A PERSPECTIVA DOS CUSTOS DE TRANSAÇÃO NA FORMAÇÃO DE REDES DE COOPERAÇÃO

Alsones Balestrin - Alessandro Porporatti Arbage

\section{RESUMO}

Este artigo tem por objetivo avançar na interpretação do fenômeno redes de cooperação no âmbito das teorias organizacionais. Parte-se do pressuposto de que a economia dos custos de transação é um dos principais motivadores para a elaboração de estruturas de governança que se situam entre o mercado e a hierarquia. Foram analisados dois casos inseridos em contextos institucionais distintos: o da Tecnópole do Futuroscope (França) e o da Rede AGIVEST (Brasil). Os resultados da pesquisa demonstram que os principais atributos das transações identificados como condicionadores dos arranjos incluem a especificidade de ativos e a incerteza. Do ponto de vista da governança, foi identificado que há a chamada governança institucional, sob responsabilidade do Estado, e a governança das atividades propriamente ditas. Além da relevância dos custos de transação, os resultados indicaram que o papel do Estado parece ser fundamental para o estabelecimento de iniciativas dessa natureza.

\section{PALAVRAS-CHAVE}

Custos de transação; redes de cooperação; pequenas e médias empresas; gestão.

\section{ABSTRACT}

This article proposes an interpretation of the phenomenon Cooperation Networks in the context of Organizational Theories. The basic assumption is that the Economy of Transaction Costs is one of the main motivating factors for the development of governance structures located between the market and the hierarchy. Two cases were analyzed and inserted into different institutional context: Futuroscope Technopole (France) and the AGIVEST Network (Brazil). The results of the research showed that the main attributes of the transactions identified as conditioning of combinations were: asset specificity and uncertainty. From a governance perspective, an institutional one was identified, under the responsibility of the State, and a governance of activities properly. Besides the relevance of transaction costs, the results indicated that the role of the State seems to be essential for the establishment of such initiatives.

\section{KEYWORDS}

Transaction costs; cooperation networks; small and medium firms; management. 
FÓRUM - SOCIOLOGIA ECONÔMICA

A PERSPECTIVA dOS CUSTOS DE TRANSAÇÃ̃O NA FORMAÇÃo DE REDES DE COOPERAÇÃO

Alsones Balestrin - Alessandro Porporatti Arbage

\section{INTRODUÇÃO}

Para as ciências sociais, o termo "rede" designa um conjunto de pessoas ou organizações interligadas direta ou indiretamente (Marcon e Moinet, 2000). Para Castells (1999), a intensidade e a freqüência da interação entre atores sociais são maiores se esses atores forem nós de uma rede do que se não pertencerem a ela. Para Nohria e Eccles (1992), existem três razões principais para o aumento do interesse sobre o estudo de redes no contexto organizacional: primeira, a emergência da nova competição, como a que está ocorrendo nos Distritos Industriais Italianos e do Vale do Silício; segunda, o surgimento das TICs (Tecnologias de Informação e Comunicação), ou seja, um conjunto de ferramentas desenvolvidas que permitem o compartilhamento de dados, como o correio eletrônico, as intranets organizacionais, a internet, o groupware, tem tornado possível uma maior capacidade de interação entre firmas dispersas; e terceira, a consolidação da análise de redes como uma disciplina acadêmica.

O amplo escopo conceitual do termo "rede" proporcionou seu interesse por um crescente número de pesquisadores das ciências sociais. Segundo um levantamento feito por Oliver e Ebers (1998), no período entre 1980 a 1996, em quatro das principais publicações sobre estudos organizacionais, observou-se um total de 158 artigos que tratavam explicitamente sobre o tema redes interorganizacionais. As publicações estavam distribuídas da seguinte forma: American Sociological Review (26 artigos), Administrative Science Quarterly (55 artigos), Academy of Management Journal (34 artigos) e Organizations Studies (43 artigos). Esses dados indicam a crescente preocupação com uma melhor compreensão sobre o fenômeno de redes, tanto no campo organizacional quanto no campo social.

Segundo Oliver e Ebers (1998) e Caglio (1998), os estudos sobre redes interorganizacionais foram conduzidos a partir de algumas correntes teóricas. A primeira, a da Economia Industrial, que identificou diferentes classes de custos de produção, como economias de escala, de escopo, de especialização e de experiência, como variáveis explicativas da eficiência das redes (Eccles, 1981; Turati, 1990; Teece, 1980). A segunda, a das Estratégias Interorganizacionais, de acordo com as quais a configuração em rede é vista como um fator estratégico no alcance e na manutenção de vantagens competitivas (Marcon e Moinet, 2000; Fayard, 2000; Jarillo, 1988). A terceira corrente, a da Teoria da Dependência de Recursos, a salientar que um dos fortes condicionantes na formação das redes interorganizacionais é a necessidade de a empresa buscar recursos complementares, sejam eles 
FÓRUM - SOCIOLOGIA ECONÔMICA

A PERSPECTIVA DOS CUSTOS DE TRANSAÇÃO NA FORMAÇÃO DE REDES DE COOPERAÇÃO

Alsones Balestrin - Alessandro Porporatti Arbage

tangíveis (tecnologia, matéria-prima etc.) ou intangíveis (informação, conhecimento, habilidades etc.) (Pfeffer e Salancik, 1978).

A quarta corrente, a da abordagem das Redes Sociais (social networks), evidenciou que a posição dos atores em uma rede influencia a organização de seus membros e apresenta forte influência nas inter-relações junto à rede (Powell et alii, 1996; Burt, 1992). A quinta, a da Teoria Institucional, constatou que as organizações buscam ganhar legitimidade em seu ambiente institucional no momento de se estruturarem em rede (DiMaggio e Powell, 1983; Grabher, 1993). E por fim a sexta corrente, representada pelas Teorias Críticas (Marxistas), que questionaram o argumento da eficiência na formação das redes e destacaram que as redes são formadas por representarem poderosos instrumentos na formação de elites e classes dominantes, bem como o exercício do poder e da dominação (Perucci e Potter, 1989; Salancik, 1995).

Por outro lado, evidênc ias empíricas sinalizam que uma das importantes motivações para a formação de redes interorganizacionais é o fato de terem demonstrado uma eficiente forma de governança das relações econômicas. Tradicionalmente, duas outras formas institucionais de governança foram freqüentemente distinguidas das redes - mercado e hierarquia. Williamson (1975), a partir dos estudos de Coase (1937), foi quem definiu o "mercado" e a "hierarquia" como os dois modos alternativos de organização das atividades econômicas. O autor considerou que uma firma contempla diferentes curvas de custos na produção de cada um dos componentes que constituem um produto final. Caso existam algumas firmas dentro do mercado produzindo o mesmo bem, a priori, a mais eficiente forma de organizar a produção consistirá de uma firma especializada na produção de cada um dos componentes do produto.

Assim, a firma especializada apresentará uma curva decrescente de custos e fornecerá para as demais firmas, as quais terão um menor custo em relação à produção desses componentes por meio da sua integração vertical. Porém, o comportamento das organizações, geralmente, não ocorre dessa forma. A explicação para tal fato, segundo Williamson (1979), é de que nas relações econômicas entre as firmas existem "Custos de Transação (CT)". Esses custos são originados pelas ineficientes transações de determinada organização com o seu mercado, o que ocorre, principalmente, pelas seguintes razões: "racionalidade limitada" do tomador de decisões, incerteza sobre o futuro e possibilidade de um "comportamento oportunista" por parte de determinados atores econômicos. Logo, a falta de confiança nas relações da empresa com o seu ambiente e a possibilidade de comportamento oportunista por parte de alguns agentes representam questões centrais na geração dos CT. 
FÓRUM - SOCIOLOGIA ECONÔMICA

A PERSPECTIVA DOS CUSTOS DE TRANSAÇÃO NA FORMAÇÃO DE REDES DE COOPERAÇÃO

Alsones Balestrin - Alessandro Porporatti Arbage

Jarillo (1988) argumenta que existe uma importante questão que não foi discutida por Williamson, de que os CT podem ser afetados pela ação consciente de um empresário, sendo essa uma das fundamentações econômicas para a "estratégia em rede". A confiança nas inter-relações entre os atores é um dos fatores que promove a redução dos CT e torna a existência das redes economicamente viáveis (Jarillo, 1988).

Uma rede interorganizacional, através da rede social de seus membros, poderá ter melhor acesso de recursos, como, por exemplo, capital e influência política. Sua intensidade de laços sociais também permite suportar um compartilhamento livre de informações entre os membros da rede, encorajando o mútuo aprendizado e inovação (Balestrin et alii, 2005). Coletivamente, as firmas integrantes da rede terão maior capacidade de adaptar-se às mudanças (Sabel 1991; Saxenian 1994). Essas vantagens são particularmente importantes dentro das características do mercado, representado pelo ritmo de evolução e custos de inovação, curtos ciclos de vida dos produtos e pressões para responder rapidamente às mudanças das necessidades dos clientes. Tais características apontam as redes como configurações que respondem às características do cenário econômico atual.

Dentro desse contexto teórico e partindo-se do pressuposto de que, na atualidade, um dos principais objetivos das empresas tem sido a busca por redução dos CT, o objetivo do artigo é avançar na interpretação do fenômeno das redes de cooperação à luz da Economia dos Custos de Transação (ECT). Em termos empíricos, o objetivo é compreender o processo de coordenação estabelecido nas redes de cooperação estudadas. Para tanto, foram analisadas duas experiências inseridas em contextos institucionais diferentes: a) A Tecnópole do Futuroscope (França); e b) A Rede AGIVEST (Brasil Rio Grande do Sul).

Além dessa introdução, o artigo está organizado em mais seis partes. Na seqüência são apresentados aspectos teóricos das redes de cooperação e da ECT. Posteriormente, são descritos os procedimentos metodológicos da pesquisa. $\mathrm{Na}$ quinta parte são descritos os casos e, por fim, são apresentadas as implicações da pesquisa e as considerações finais.

\section{REDES DE COOPERAÇÃO}

As diversas tipologias de redes interorganizacionais têm provocado alguma ambigüidade no próprio entendimento do termo. Essa questão foi objeto da análise de Castells (1999), com base no argumento de que as redes interorganizacionais aparecem sob diferentes formas, em diferentes contextos e a partir 
FÓRUM - SOCIOLOGIA ECONÔMICA

A PERSPECTIVA DOS CUSTOS DE TRANSAÇÃO NA FORMAÇÃO DE REDES DE COOPERAÇÃO

Alsones Balestrin - Alessandro Porporatti Arbage

de expressões culturais diversas. Buscando uma melhor compreensão sobre a diversidade de tipologias de redes interorganizacionais, Marcon e Moinet (2000) apresentaram um gráfico denominado de "mapa de orientação conceitual" (conforme Figura 1), indicando, a partir de quatro quadrantes, as principais dimensões sobre as quais as redes são estruturadas.

Figura 1. Mapa de orientação conceitual: modo de gerenciamento e formação de elos

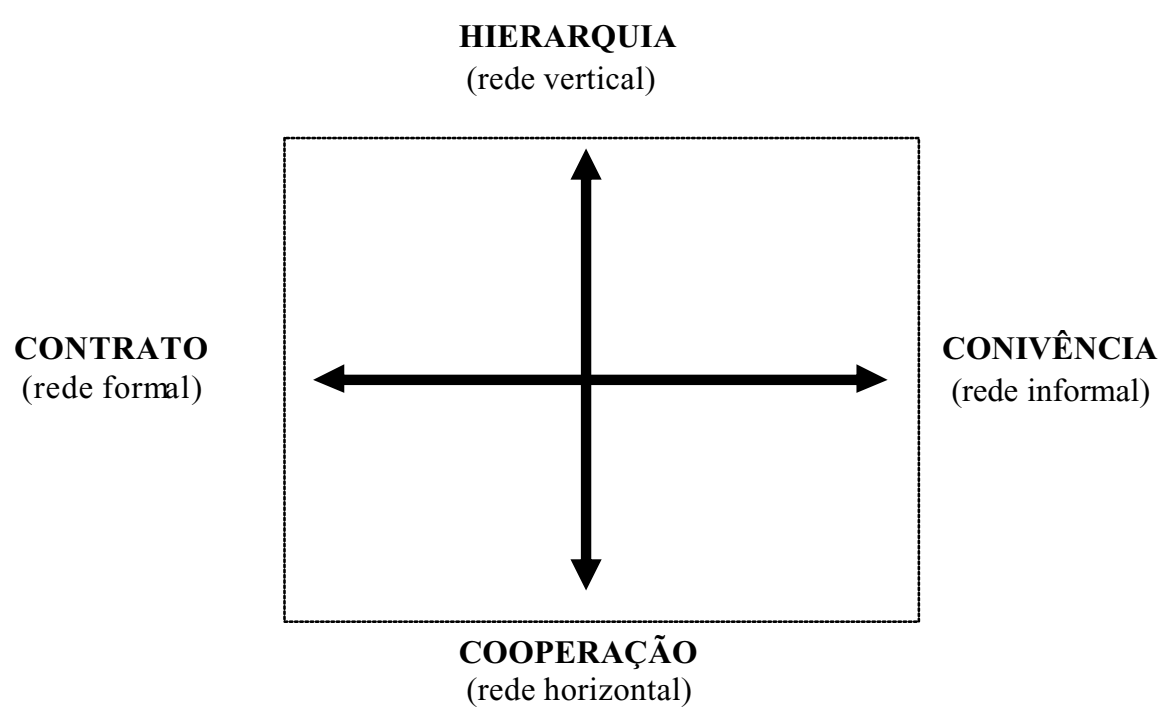

Fonte: Elaborada pelos autores a partir do modelo de Marcon e Moinet (2000)

Segundo as orientações desse mapa conceitual, o eixo vertical relaciona-se com a natureza dos elos gerenciais estabelecidos entre os atores da rede. Esses elos podem representar uma relação de cooperação (no caso de uma rede horizontal, como as redes de cooperação entre Pequenas e Médias Empresas - PME), ou representar um grau de ligação hierárquico (no caso de uma rede vertical, como uma rede do tipo matriz e filial).

Já o eixo horizontal representa o grau de formalização estabelecido nas relações entre os atores. Esse grau pode mover-se de uma conivência informal entre os atores (no caso de relações de amizade, afinidade e parentesco, tal como ocorrem nas associações, nos clubes, nas redes de amigos) até relações formalmente estabelecidas por contratos entre as partes (no caso de contratos jurídicos, como ocorre na formação de joint-ventures). Deve-se destacar que, em cada um dos diversos pontos do quadrante, poderá ser encontrado um tipo particular de configuração de rede, elucidando, assim, a ampla diversidade de tipologias de redes interorganizacionais. Logo, de acordo com as orientações da Figura 1, as redes podem ser classificadas em quatro grupos: a) redes verticais - dimensão da hierarquia; b) 
FÓRUM - SOCIOLOGIA ECONÔMICA

\section{A PERSPECTIVA DOS CUSTOS DE TRANSAÇÃO NA FORMAÇÃO DE REDES DE COOPERAÇÃO}

Alsones Balestrin - Alessandro Porporatti Arbage

redes horizontais - dimensão da cooperação; c) redes formais - dimensão contratual; d) redes informais - dimensão da conivência.

As redes horizontais (objeto de estudo no presente artigo), também denominadas de "redes de cooperação", constituídas por empresas que guardam cada uma sua independência, mas que optam por desenvolver atividades específicas de maneira conjunta para atingir determinados objetivos comuns. Existe uma grande heterogeneidade de redes horizontais fundamentadas na cooperação, como por exemplo: os consórcios de empresas, as redes de cooperação entre PME, as redes de lobbying, bem como as relações de cooperação entre empresas que ocorrem junto às tecnópoles e aos clusters. As relações interorganizacionais estabelecidas nesses arranjos são complexas, nas quais os atores, muitas vezes concorrentes, escolhem cooperar dentro de certo domínio. Assim, as redes favorecem a concentração de esforços sem privar a liberdade de ação estratégica de seus membros.

No entanto, mesmo com a reconhecida relevância da dimensão da cooperação nas relações interorganizacionais, sobretudo no caso das PME, Human e Provan (1997) destacam a existência de apenas alguns estudos isolados sobre as redes de cooperação. Para Perrow (1992), o fenômeno das redes tem sido pouco estudado pelas teorias clássicas, argumentando que o modelo de produção representado pela grande firma integrada, defendida originalmente por Chandler (1977), tornou-se um modelo declinante frente às necessidades contemporâneas de flexibilização. Perrow (1992) acrescenta que o problema da teoria de Chandler (1977) foi negligenciar completamente o papel atribuído à cooperação nos modelos econômicos. O autor acrescenta que as teorias clássicas necessitam ser questionadas, pois negligenciaram o poder econômico de três fatores que ajudam a explicar o sucesso das redes: as economias de escala por meio de redes, a confiança e a cooperação que coexistem com a competição e o estado de bem-estar social causado pelo aumento da eficiência de setores industriais regionais.

Outro fator apresentado por Perrow (1992) é o fato de que as redes de cooperação são geralmente inseridas em um ambiente institucional que é essencial para sua sobrevivência e para sua economia, o qual abrange incentivos de governos locais e regionais, serviços educacionais, associações comerciais que fornecem informação econômica, treinamento e serviços de marketing. Esse ambiente, comumente encontrado nos distritos industriais italianos, apresenta uma forte contribuição para o desenvolvimento de regiões e para o próprio progresso das empresas. 
FÓRUM - SOCIOLOGIA ECONÔMICA

A PERSPECTIVA DOS CUSTOS DE TRANSAÇÃO NA FORMAÇÃO DE REDES DE COOPERAÇÃO

CUSTOS DE TRANSAÇÃO

Alsones Balestrin - Alessandro Porporatti Arbage

Influenciados pelos pioneiros trabalhos de Commons (1924; 1931) e Coase (1937), um significativo número de pesquisadores tem dedicado atenção ao estudo do comportamento das organizações na interface existente entre mercado e hierarquia. Ao menos dois grupos de pesquisas podem ser distinguidos dentro desse campo do conhecimento: a ECT e a Teoria da Agência. Dessas linhas de pesquisa, a Nova Economia Institucional, mais precisamente a ECT, é seguramente a mais elaborada cons trução teórica explicativa dos arranjos existentes na realidade organizacional.

O conceito de transação foi definido por Williamson (1975) como estando presente sempre que há a transferência de um bem através de uma interface tecnologicamente separável. Para Rezende (1999), os Custos de Transação (CT) são os custos para se gerenciar o sistema econômico por meio da identificação, explicação e atenuação dos riscos contratuais. Barzel (1997) define CT como os custos relacionados com a transferência, captura e proteção dos direitos de propriedade. Já Eggerstsson (1990) pondera que, em geral, os CT são os custos que aparecem quando os indivíduos trocam direitos de propriedade de ativos econômicos e reforçam seus direitos exclusivos.

$\mathrm{Na}$ literatura especializada há uma plêiade de definições do que são CT. No entanto, a que parece ser a mais pertinente tendo em vista a abrangência do conceito é a de Arrow (1969). O autor definiu que os CT são os custos associados à administração do sistema econômico. Segundo o autor, enquanto os custos de produção formam a variável de que se ocupou detidamente a Economia Neoclássica, os CT caracterizam-se por ser aqueles que ocorrem ao se colocar em funcionamento o sistema econômico.

Partindo-se dessa noção, e seguindo a literatura especializada, identifica-se um grupo de elementos que se relacionam direta e indiretamente com os CT. São eles: oportunismo, racionalidade, incerteza, risco, especificidade de ativos, freqüência das transações e ambiente institucional. Vejamos cada um desses elementos a seguir.

A idéia central do oportunismo relaciona-se ao padrão de orientação que o indivíduo estabelece para a busca do seu próprio interesse e que termina por refletir-se no padrão de relacionamento estabelecido entre os agentes econômicos. Williamson sustenta que o oportunismo é a busca pelo interesse próprio com dolo.

Segundo Williamson (1985), ao longo da Teoria Econômica se distinguem três níveis de racionalidade: uma forma forte, considerada a partir dos esforços de maximização; uma forma semiforte, caracterizada com a racionalidade limitada; e a forma débil ou mais fraca, que se denomina 
FÓRUM - SOCIOLOGIA ECONÔMICA

A PERSPECTIVA DOS CUSTOS DE TRANSAÇÃO NA FORMAÇÃO DE REDES DE COOPERAÇÃO

Alsones Balestrin - Alessandro Porporatti Arbage

de racionalidade orgânica. A racionalidade limitada caracteriza-se como sendo uma modalidade em que se supõe que os atores econômicos são intencionalmente racionais, mas, devido às limitações de natureza cognitiva, apenas parte do conjunto de informações consegue ser obtido e processado individualmente para a tomada de decisão mais adequada.

A incerteza relaciona-se ao desconhecimento dos agentes de elementos relacionados ao ambiente econômico, institucional e comportamental, que exercem algum tipo de influência na gestão dos negócios. Segundo Arbage (2004), há incerteza de três naturezas: incerteza primária, incerteza secundária e incerteza comportamental ou conductista.

A incerteza primária refere-se ao desconhecimento de informações relacionadas ao ambiente institucional, como, por exemplo, alterações em determinados padrões comportamentais dos consumidores. A incerteza secundária caracteriza-se pelo desconhecimento, geralmente por falta de comunicação adequada, de elementos de natureza estratégica importantes para os agentes que se relacionam em torno de uma transação. Por seu turno, a incerteza comportamental é um subtipo da categoria analítica vinculada aos aspectos estratégicos e que se relacionam com o oportunismo: é a incerteza que decorre do desconhecimento do padrão de comportamento dos parceiros com os quais uma determinada organização realiza suas transações.

$\mathrm{O}$ risco se associa positivamente com os dois elementos referidos anteriormente, quer dizer, com o oportunismo e a racionalidade. Não fossem esses aspectos, não haveria o risco de as transações não ocorrerem da forma como foram planejadas, nem tampouco de que algo importante deixasse de ser previsto antecipadamente. Quanto maior o nível de oportunismo, assim como quanto menos informações estejam disponíveis para a tomada de decisão, aumenta o risco de que as "coisas não saiam como planejadas". No entanto, como uma medida do risco pode ser obtida através de uma distribuição de probabilidade do evento, esse aspecto pode ser inserido no planejamento das organizações.

A questão da especificidade dos ativos é um dos pontos importantes da teoria dos CT e fonte de atritos. O ponto-chave destacado é a maior ou menor possibilidade de utilização alternativa de um ativo relacionado aos custos envolvidos em um processo de produção. Quanto maior a especificidade de um ativo, menor tende a ser a possibilidade de utilização alternativa do mesmo. As partes de uma transação podem exigir investimentos de propósitos gerais ou específicos. As transações que exigem investimentos específicos implicam custos de transação maiores devido exatamente ao fato de que sua reutilização não ocorre de forma automática e sem perdas. 
FÓRUM - SOCIOLOGIA ECONÔMICA

A PERSPECTIVA DOS CUSTOS DE TRANSAÇÃO NA FORMAÇÃO DE REDES DE COOPERAÇÃO

Alsones Balestrin - Alessandro Porporatti Arbage

Por fim, em relação à freqüência das transações o que ocorre é que, quando se exige algum tipo de ativo específico, deve haver uma contrapartida em termos de freqüência de transações para que o investimento realizado seja mais rapidamente amortizado. Não é razoável que a produção que exige um investimento idiossincrático possa ser sustentada por um padrão de transações de baixa freqüência.

\section{METODOLOGIA DA PESQUISA}

Os casos selecionados para a pesquisa foram a Tecnópole do Futuroscope, localizada m Região de Poitiers (França), e a Rede AGIVEST (Associação Gaúcha de Indústrias do Vestuário), localizada na Região Sul do Brasil. A escolha de tais casos deveu-se às seguintes motivações: a) os casos são formados, na sua maioria, por Pequenas e Médias Empresas (PME), importante foco de estudo dos pesquisadores; b) os casos apresentam similar configuração, ou seja, vários atores institucionais (empresas, universidade, poder público etc.) geograficamente próximos e articulando ações conjuntas; c) são situações que resultaram de políticas públicas e privadas com objetivos vinculados ao desenvolvimento regional; e d) os casos buscam criar um ambiente de sinergia a fim de potencializar a cooperação para o desenvolvimento das empresas.

Para a pesquisa, adotourse uma abordagem complementar de evidências, em vez de propriamente uma replicação comparativa de casos. Apesar da diversidade de resultados que os casos possam suscitar, o que demanda um maior cuidado e complexidade de análise, acredita-se que eles possibilitem cobrir de maneira mais satisfatória a heterogeneidade e a variabilidade dos elementos conceituais envolvidos nesse estudo.

A operacionalização do estudo de campo ocorreu da seguinte forma. No caso da Tecnópole do Futuroscope, o estudo de campo foi conduzido no mês de junho de 2003. A pesquisa foi direcionada às PME da Tecnópole do Futuroscope. Foram realizadas 13 entrevistas com dirigentes de empresas selecionadas a partir de um total de 50 empresas do segmento de internet e multimídia. A seleção das empresas foi aleatória, tendo sido realizada a partir do cadastro oficial de empresas disponibilizado pelo CG (Conseil Général de la Vienne - órgão do poder público regional e responsável pela gestão da Tecnópole).

Quanto à AGIVEST, o estudo de campo foi conduzido no mês de novembro de 2003, a partir de entrevistas realizadas com o presidente da rede e com quatro dirigentes de PME pertencentes à AGIVEST. Em ambos os casos, as entrevistas tiveram uma duração aproximada de 40 minutos. O 
FÓRUM - SOCIOLOGIA ECONÔMICA

A PERSPECTIVA DOS CUSTOS DE TRANSAÇÃO NA FORMAÇÃO DE REDES DE COOPERAÇÃO

Alsones Balestrin - Alessandro Porporatti Arbage

pesquisador utilizou um roteiro de entrevista semi-estruturado, elaborado a partir do Quadro 1, com o objetivo de apresentar uma seqüência lógica das questões aos entrevistados.

Deve-se destacar que a definição do número de entrevistas não teve qualquer relação com a representatividade estatística. A intenção foi buscar um nível de evidências empíricas suficientes frente à problemática de pesquisa. Diante disso, o número de entrevistas na Tecnópole teve de ser maior em função da dificuldade de pesquisa proporcionada pelo contexto cultural não-familiar para os pesquisadores.

Quadro 1. Operacionalização da Pesquisa

\begin{tabular}{|c|c|}
\hline Elementos Conceituais & Dimensões de Análise \\
\hline $\begin{array}{c}\text { Características dos arranjos } \\
\text { pesquisados }\end{array}$ & $\begin{array}{c}\text { Tamanho, idade, tipo de produto, nível tecnológico e ambiente institucional. } \\
\text { Elementos geradores de CT }\end{array}$ \\
$\begin{array}{c}\text { Racionalidade (forte, limitada ou orgânica); incerteza; risco; oportunismo; } \\
\text { especificidades dos ativos (físicos, marca, locacional ou de conhecimento) e } \\
\text { freqüência das transações. }\end{array}$ \\
\hline Governança & Forma de governança encontrada no arranjo estudado. \\
\hline
\end{tabular}

Para a interpretação dos resultados, as entrevistas foram transcritas e, posteriormente, os relatórios foram submetidos à análise de conteúdo. Os resultados das entrevistas e das observações realizadas foram confrontados com os elementos conceituais. Conforme orientações de Yin (2001) e Wacheux (1996), esse procedimento visa ao avanço na compreensão do fenômeno em estudo e das implicações teóricas. Em um primeiro momento, os dois casos foram analisados individualmente; depois, em conjunto. $\mathrm{O}$ foco esteve na identificação dos elementos semelhantes e divergentes que poderiam refletir-se nas conclusões da pesquisa.

\section{EVIDÊNCIAS EMPÍRICAS}

\section{O caso da Tecnópole do Futuroscope}

A Tecnópole foi criada há 20 anos com o objetivo de desenvolver uma região caracterizada pela economia agroalimentar e pelo setor terciário mais voltado à administração pública. Diferentemente de 
FÓRUM - SOCIOLOGIA ECONÔMICA

\section{A PERSPECTIVA DOS CUSTOS DE TRANSAÇÃO NA FORMAÇÃO DE REDES DE COOPERAÇÃO}

Alsones Balestrin - Alessandro Porporatti Arbage

outras Tecnópoles, o referido projeto apresenta uma proposta original ao estar ancorado em três pilares - lazer, negócio e pesquisa - e estar localizado em um espaço geográfico de 80 hectares.

Do ponto de vista do contexto institucional em que a Tecnópole está inserida, pode-se identificar que existem múltiplos atores, como empresas localizadas nos condomínios (área construída para hospedar empresas já constituídas), empresas localizadas na incubadora (pépinière d'entreprises), parque da imagem, CG, Universidade de Poitiers, CNED (Centre National d'Enseignement à Distance), hotéis, entre outros institutos de pesquisa e associações, conforme pode ser observado na Figura 2.

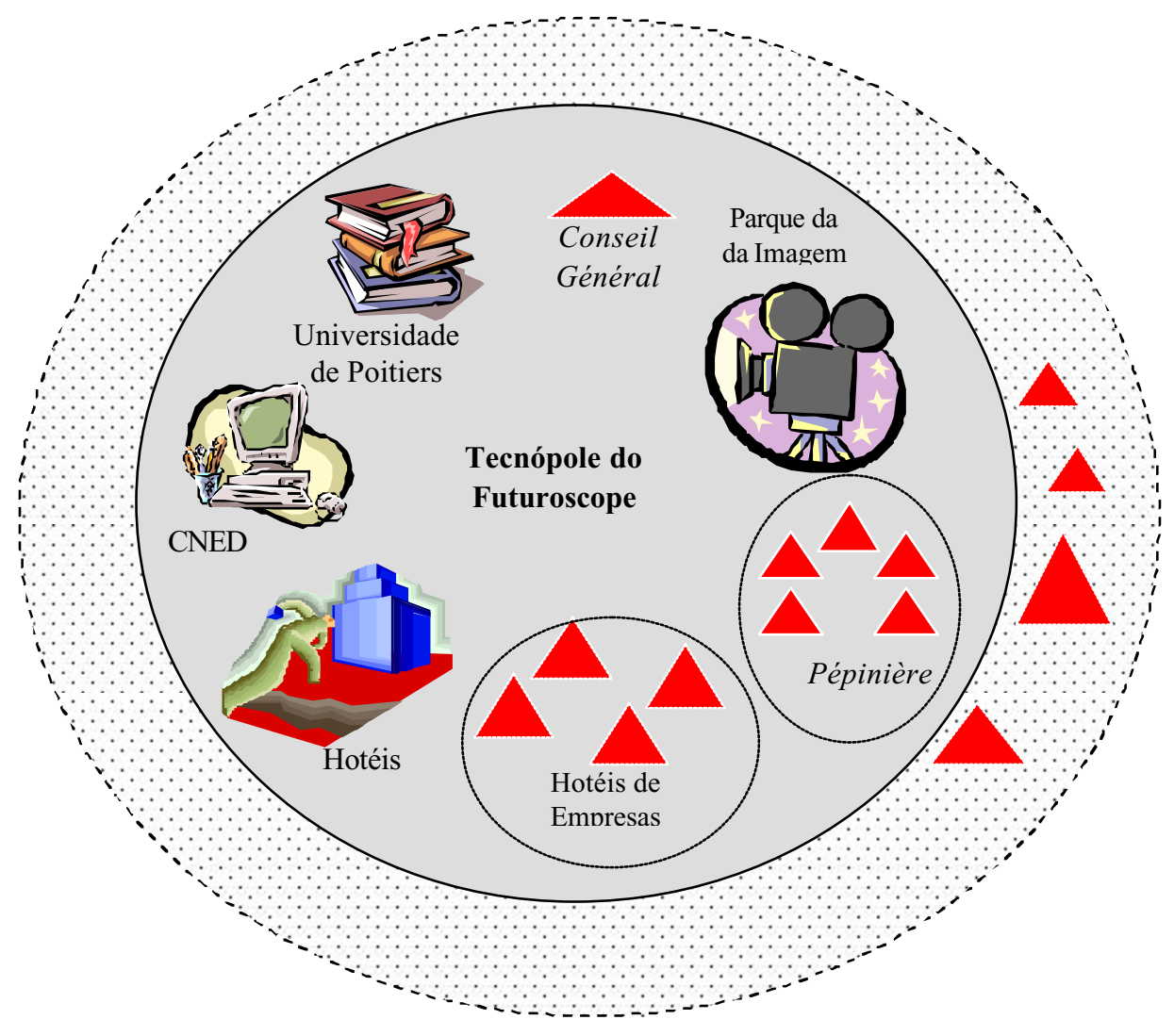

Figura 2. Contexto Institucional da Tecnópole

A Tecnópole posiciona-se como uma referência nacional em abrigar empresas do segmento de TIC. Existem aproximadamente 100 empresas; entre elas, aproximadamente 50 PMEs dedicamse exclusivamente ao desenvolvimento de soluções multimídia, ao tratamento da informação e às aplicações para internet. O parque do Futuroscope é reconhecido como o primeiro parque de imagem 
FÓRUM - SOCIOLOGIA ECONÔMICA

A PERSPECTIVA DOS CUSTOS DE TRANSAÇÃO NA FORMAÇÃO DE REDES DE COOPERAÇÃO

Alsones Balestrin - Alessandro Porporatti Arbage

da Europa e o segundo parque de atrações na França, responsável por trazer para a região aproximadamente 2,5 milhões de turistas ao ano. No parque da imagem podem ser apreciadas atrações como filmes em três dimensões e realidade virtual em cinemas com efeitos especiais.

A dimensão da pesquisa está muito presente no conceito da Tecnópole. Ela conta com aproximadamente 700 pesquisadores e mais de 2 mil estudantes em centros de ensino e pesquisa. $\mathrm{Na}$ região se encontram a Escola Superior de Comércio e Gerenciamento (ESCEM), a Escola Superior de Engenharia de Poitiers (ESIP), a Escola Nacional Superior de Mecânica e Aeronáutica (ENSMA) e o Instituto de Comunicação e Novas Tecnologias (ICOMTEC).

Todas as atividades de lazer e pesquisa foram concebidas para atrair atores e criar uma “atmosfera de inovação". Existem 50 mil metros quadrados em espaços disponíveis, além de uma infraestrutura para acolher e acompanhar as empresas. Alguns exemplos são a incubadora de empresas, que oferece as condições para os portadores de projetos criarem seus empreendimentos, aliado ao centro de empresas e de inovação (Centre d'entreprises et d'innovation - CEI), que coloca à disposição dos empreendedores uma série de serviços de acompanhamento e de consultoria.

Para entender a dinâmica das relações interorganizacionais foi necessário identificar os elementos motivadores que fizeram com que as empresas se inserissem na Tecnópole. Segundo a pesquisa, os principais aspectos, em ordem decrescente de importância, foram: a) imagem positiva que o consumidor passa a fazer da empresa em virtude de a mesma ter-se agregado à Tecnópole (legitimidade no mercado); b) infra-estrutura disponível; c) maior possibilidade de cooperação; e d) compartilhamento de informações, conhecimentos técnicos e de mercado.

A imagem de "cidade do futuro", com uma arquitetura particular (futurista) e com atrações no parque da imagem, juntamente com o ganho de eficiência viabilizado pelo baixo custo da estrutura física e de serviços, foram indicados como os mais significativos motivos que levaram as empresas a se inserirem na Tecnópole. Os outros aspectos citados como importantes, mas em uma escala bem abaixo dos anteriores, foram: a possibilidade de usufruir uma infra-estrutura moderna de telecomunicações, a própria posição geográfica da Tecnópole, assim como os aspectos relacionados com a troca de informações (conhecimentos técnicos, comerciais e cooperação informacional em linhas gerais).

Do ponto de vista da coordenação, identificoutse que a mesma é estabelecida por um órgão do poder público regional responsável pela promoção e gestão pública da Região da Vienne, na França: Conseil General (CG). É de responsabilidade do CG a construção da infra-estrutura onde as empresas serão instaladas. Cabe também a uma comissão dirigida pelo CG o processo de seleção das empresas para se instalar na Tecnópole, através de critérios estabelecidos previamente. A gestão da incubadora é 
FÓRUM - SOCIOLOGIA ECONÔMICA

A PERSPECTIVA DOS CUSTOS DE TRANSAÇÃO NA FORMAÇÃO DE REDES DE COOPERAÇÃO

Alsones Balestrin - Alessandro Porporatti Arbage

de responsabilidade do CEI, órgão mantido pelo poder público e responsável pela promoção e pelo fomento de empresas de caráter inovador junto à Tecnópole.

Um fator de destaque da Tecnópole são as atividades que envolvem a acolhida e o suporte aos empreendedores portadores de projetos inovadores. Essas atividades são desenvolvidas principalmente pelo CEI e pela Incubadora Tecnológica. Atualmente, a Incubadora acolhe duas dezenas de empresas para uma duração máxima de 18 meses. Muitos dos empresários entrevistados reconheceram que a Incubadora apresenta uma grande contribuição no acompanhamento das empresas em seus estágios iniciais. Certamente, as atividades desses centros de acompanhamento e criação de novas empresas fazem da Tecnópole um local de referência na criação de empreendimentos inovadores.

No entanto, alguns problemas foram identificados pelos empresários na composição da Tecnópole. Segundo eles, mesmo existindo exemplos de complementaridade, alguns empresários manifestaram um sentimento de "solidão", apesar de estarem próximos uns aos outros, indicando problemas centrais que levam à dificuldade de engajar maiores ações coletivas e de cooperação.

Nas entrevistas, evidenciou-se que o problema da falta de interação entre as empresas é resultado da limitada existência de situações que possam facilitar os encontros entre os empresários. A pesquisa demonstrou um problema capital, pois, ao investir vultuosos recursos para reunir diversos atores em um mesmo local geográfico, o objetivo maior é criar um ambiente de aprendizado e proporcionar condições favoráveis para um contexto de sinergia e de inovação. Porém, essa dinâmica não nasce pelo simples fato de se colocar as empresas juntas; é necessário organizar essa proximidade e articular a interação entre os atores.

Muitas foram as sugestões para a Tecnópole tornar-se um pólo de produção e difusão de inovações tecnológicas. Entre as principais pôde-se identificar: aproximar as pequenas e médias empresas dos laboratórios de pesquisa das universidades; criar espaços e momentos formais e informais para oportunizar o encontro dos empresários; articular reuniões temáticas em torno de projetos de interesse comum; fazer uma cartografia das competências e das necessidades de empresas e de instituições presentes na Tecnópole para facilitar o engajamento em projetos de cooperação.

\section{O caso da AGIVEST}

A AGIVEST é formada por 35 pequenas indústrias do setor têxtil e do segmento de confecções. Localiza-se na região de Ijuí, no Rio Grande do Sul, e faz parte do Programa Redes de Cooperação do Governo do Estado. Sua constituição ocorreu em setembro de 2001 com o principal objetivo de 
FÓRUM - SOCIOLOGIA ECONÔMICA

A PERSPECTIVA DOS CUSTOS DE TRANSAÇÃO NA FORMAÇ̃̃o DE REDES DE COOPERAÇÃO

Alsones Balestrin - Alessandro Porporatti Arbage

proporcionar melhores condições de competitividade para as pequenas empresas participantes por meio do fortalecimento da cooperação.

As empresas caracterizam-se por serem pequenas - são compostas, na média, por seis empregados, utilizando-se basicamente de mão-de-obra dos familiares e de pessoas próximas. Os fundadores e os colaboradores apresentam uma modesta qualificação técnica, tanto em termos de gestão quanto no desenvolvimento de produtos, resultado de uma origem artesanal, característica comum no segmento de confecções da região sul do país. São empresas com uma média de sete anos de constituição. Todas começaram suas atividades em pequenos ateliês, justamente por ser uma atividade que demanda baixos investimentos em capital fixo e requer pouca complexidade nos processos de produção. As empresas pesquisadas trabalham com as seguintes linhas de produtos: artigos esportivos, uniformes profissionais, linha íntima feminina e malhas em geral.

A pesquisa identificou que a maioria das empresas tem por objetivo a especialização em uma determinada linha de produto. Esse fato por um lado é salutar, na medida em que possibilita a diminuição da concorrência entre as empresas, e, por outro, permite a ampliação do mix de produtos a serem produzidos no âmbito da AGIVEST. Além disso, tal especialização pode apresentar conseqüências indesejáveis, como, por exemplo, a impossibilidade de barganhar preços em grandes volumes de compra junto aos fornecedores, pois poucos serão os suprimentos comuns a todas as empresas.

O desenvolvimento tecnológico da rede como um todo é limitado, sobretudo pelos escassos recursos para investir em tecnologia de produção, mão-de-obra mais qualificada e para a contratação de estilistas mais capacitados. Essas carências estão sendo gradativamente superadas pela ação coletiva da rede, como, por exemplo, a contratação de renomado estilista para elaboração de uma coleção nacional. Segundo o entendimento dos entrevistados, torna-se difícil para os empresários, individualmente, mudar a concepção de design dos produtos de um mercado local para um mercado nacional e acompanhar as tendências deste segmento mais atentamente.

Do ponto de vista do contexto institucional em que a AGIVEST está inserida, pode-se identificar que há um considerável conjunto de atores governamentais e não governamentais que se relacionam com a Rede, conforme Figura 3. O governo estadual, as prefeituras municipais, a UNIJUI (Universidade de Ijuí), o SEBRAE (Serviço Brasileiro de Apoio às Micro e Pequenas Empresas) e as associações industriais municipais são os agentes que exercem forte apoio para o desenvolvimento e consolidação da AGIVEST. 
FÓRUM - SOCIOLOGIA ECONÔMICA

A PERSPECTIVA DOS CUSTOS DE TRANSAÇÃO NA FORMAÇÃO DE REDES DE COOPERAÇÃO

Alsones Balestrin - Alessandro Porporatti Arbage

O Governo do Estado, por meio da SEDAI (Secretaria de Desenvolvimento e de Assuntos Internacionais), se constituiu no ator de maior relevância na formação da AGIVEST. Este órgão firmou convênios com a UNIJUÍ para atividades de consultoria e implantação da Rede. A UNIJUÍ também promoveu seminários para a comunidade com palestrantes nacionais e internacionais com o objetivo de proporcionar maior reflexão sobre as oportunidades e os desafios em trabalhar em uma configuração de rede. O SEBRAE atendeu a demandas pontuais por parte das pequenas e médias empresas, como, por exemplo, financiar a participação em feiras, prestar consultorias de promoção da marca AGIVEST e nos processos de produção. Já, as prefeituras e as associações industriais municipais, apoiaram os empresários em ações para a promoção das empresas visando a consolidar a rede ao disponibilizar gratuitamente um espaço para a administração da AGIVEST.

Figura 3. Contexto Institucional da AGIVEST

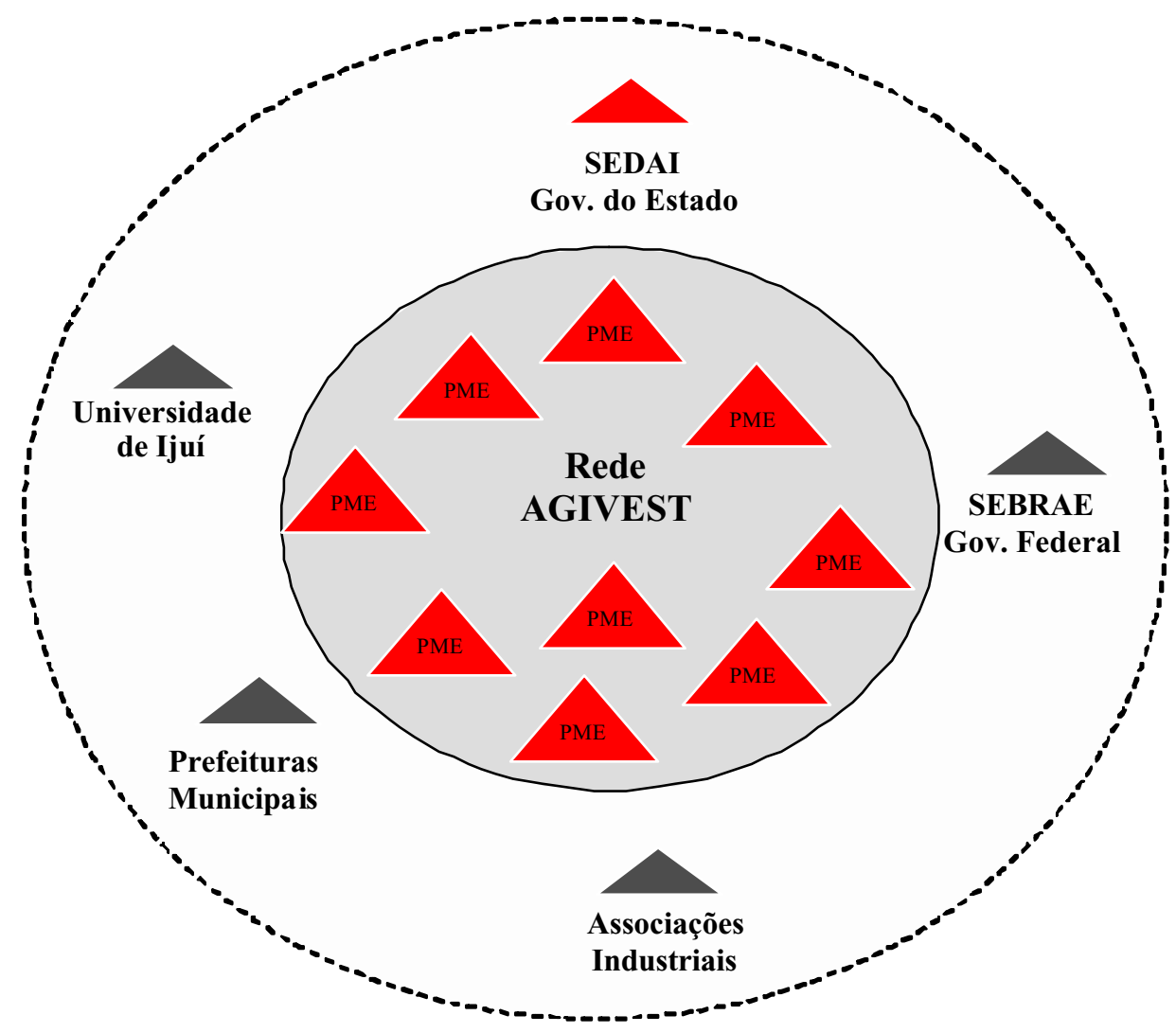

Assim como no caso da Tecnópole, para entender a dinâmica das relações interorganizacionais estabelecidas entre as empresas e demais instituições foi necessário identificar os elementos motivadores que fizeram com que as empresas se inserissem na AGIVEST. Segundo os resultados da 
FÓRUM - SOCIOLOGIA ECONÔMICA

\section{A PERSPECTIVA DOS CUSTOS DE TRANSAÇÃO NA FORMAÇÃO DE REDES DE COOPERAÇÃO}

Alsones Balestrin - Alessandro Porporatti Arbage

pesquisa, os principais aspectos que motivaram as empresas a participar de uma rede de cooperação foram: a) a necessidade de melhorar as condições de competitividade; e b) a busca de cooperação e reciprocidade.

Para os dirigentes entrevistados, a principal motivação para participar da AGIVEST foi a tentativa de melhorar as condições de sobrevivência, pois as empresas estavam encontrando dificuldades para obter um posicionamento competitivo mais favorável em um mercado bastante dinâmico, como é o caso do segmento de confecções. A necessidade maior recaiu sobre a possibilidade de acessar recursos específicos, tais como técnicas de produção, conhecimentos de mercado (representantes, clientes, fornecedores, tecnologia) e conhecimentos de gestão. A tentativa de estabelecer um ambiente que possibilitasse cooperação e o estabelecimento de maior reciprocidade nas relações entre as organizações representaram uma motivação especial para a participação na AGIVEST.

Observou-se nas entrevistas que as relações interfirmas minimizam o "sentimento de solidão" que os dirigentes sentiam anteriormente. A expressão "sair do isolamento", também utilizada, significa a possibilidade de socializar os problemas e as experiências. Da mesma forma, alguns manifestaram que o objetivo de buscar novos conhecimentos está sendo alcançado. Também foi citado o fato de que participar de uma rede pode aumentar as vendas.

Dentro desse contexto, identificou-se que os primeiros esforços dos atores-chave e as estratégias iniciais estabelecidas para o conjunto de PME que se inseriram na AGIVEST foram atender à necessidade de formação gerencial dos administradores e buscar uma padronização dos processos industriais, sendo que o aumento nas vendas foi deixado em um segundo momento. Uma das unanimidades entre os entrevistados foi a importância atribuída ao acesso de conhecimentos a partir da entrada na rede. A principal fonte de socialização de conhecimentos decorreu de uma maior comunicação que passou a ocorrer entre os empresários; porém, outras fontes de troca de conhecimentos foram observadas, como presença em feiras, cursos de formação, palestras, consultorias e visitas a outras empresas da rede.

Do ponto de vista da coordenação, a AGIVEST apresenta uma estrutura de gestão composta por um presidente e um vice-presidente. O órgão máximo para a tomada de decisões é a Assembléia dos Associados, instância institucional com amplos poderes deliberativos. A assembléia tem um papel relevante para a governança da rede, sendo realizada com a presença de um representante por empresa associada. A rede também possui instrumentos legais que ditam normas de comportamento, tais como código de ética, estatuto e regimento interno. Esses instrumentos são utilizados para garantir direitos e 
FÓRUM - SOCIOLOGIA ECONÔMICA

A PERSPECTIVA DOS CUSTOS DE TRANSAÇÃO NA FORMAÇ̃̃o DE REDES DE COOPERAÇÃo

Alsones Balestrin - Alessandro Porporatti Arbage

prevenir possíveis comportamentos indesejáveis por parte dos empresários. Para facilitar a governança, a AGIVEST conta atualmente com uma consultoria na área de marketing e produção.

No ano de 2003, 9 das 44 empresas que faziam parte saíram da AGIVEST. Dois fatos foram apontados como os principais responsáveis. O primeiro foi decorrente da existência de assimetria de tamanho e de desenvolvimento tecnológico das empresas, uma vez que as empresas menores não conseguiam acompanhar as maiores nos níveis de investimentos demandados para as ações conjuntas. Um outro fator apontado pelos entrevistados foi a tentativa de obtenção de resultados financeiros imediatos por parte de alguns empresários.

Para aprimorar o desenvolvimento gerencial dos empresários, o governo do Estado proporcionou cursos de capacitação gerencial. Nesses cursos, os empresários desenvolvem conceitos e técnicas de gestão empresarial. Tal aprendizado gerencial é relevante no momento em que as empresas trabalham com uma marca única e, assim, os padrões de processos de produção e de gestão devem ser observados a fim de garantir a qualidade exigida para os produtos da marca. Outro exemplo de situações de construção de conhecimento na AGIVEST foi a elaboração de um planejamento estratégico, realizado de forma participativa por todos os empresários.

\section{IMPLICAÇÕES DA PESQUISA}

A análise do caso da Tecnópole evidenciou algumas questões interessantes do ponto de vista dos CT. Em primeiro lugar, a criação de um "Centro de irradiação de conhecimentos em tecnologias de informação" ou o que os entrevistados salientaram como "atmosfera de inovação" se insere exatamente no que a literatura considera como "conhecimentos idiossincráticos". Esse é um ativo específico, e a construção de um espaço que permita a emergência e o compartilhamento desse ativo visa a reduzir os CT para os agentes que utilizam conjuntamente esse recurso de produção.

Da mesma forma, a interpretação das entrevistas indicou que o aspecto mais relevante para que as empresas optem por fazer parte da Tecnópole foi a construção de uma imagem positiva junto aos consumidores. A imagem de "cidade do futuro" associada à Tecnópole é um elemento redutor dos CT do ponto de vista dos consumidores, na medida em que se pressupõe que uma empresa, ao fazer parte de uma rede com tais características, de alguma forma está demonstrando aos consumidores que é uma empresa moderna e de qualidade. Assim sendo, considera-se que a "reputação" é um ativo que propicia legitimidade no mercado, sendo, portanto, um ativo específico. 
FÓRUM - SOCIOLOGIA ECONÔMICA

A PERSPECTIVA DOS CUSTOS DE TRANSAÇÃO NA FORMAÇÃO DE REDES DE COOPERAÇÃO

Alsones Balestrin - Alessandro Porporatti Arbage

A questão da cooperação entre as organizações pode ser interpretada à luz da minimização da incerteza secundária entre os agentes. Considera-se como incerteza secundária a que decorre do desconhecimento do comportamento estratégico dos fornecedores e clientes diretos da empresa. O compartilhamento de informações, conhecimentos técnicos e de mercado são exatamente a tentativa dos agentes em ampliar a "racionalidade" e, conseqüentemente, minimizar o que a literatura considera como "racionalidade limitada dos agentes econômicos".

Do ponto de vista da governança das ações organizacionais, identificourse que ela é subdividida em governança institucional e tecnológica. A primeira é realizada pelo Conseil Général, órgão do poder público regional, que fornece infra-estrutura locacional e de serviços e realiza o processo de seleção das empresas. A governança tecnológica é realizada pelo Centre d'entreprises et d'innovation (CEI), também um órgão do poder público, e que tem por função a gestão da inovação na Tecnópole.

A análise do caso da AGIVEST permitiu reforçar algumas das evidências já percebidas no caso anterior. O primeiro aspecto a ressaltar é a motivação, tanto por parte do governo do Estado quanto das PME em participar da rede, ou seja, a necessidade de estabelecer melhores condições de competitividade. O programa Redes de Cooperação, desenvolvido pelo poder público Estadual, identificou dificuldades competitivas para uma série de PME em diversos setores das atividades econômicas. A interpretação das entrevistas indicou que os dois aspectos mais relevantes para as empresas optarem por fazer parte da AGIVEST foram: a) buscar melhores condições de competitividade coletiva; e b) estabelecer maior nível de cooperação e reciprocidade nas relações econômicas com os parceiros comerciais.

Para melhorar a competitividade, foi citado o desenvolvimento de técnicas de produção, o compartilhamento de informações de mercado e melhorias no campo gerencial. O desenvolvimento de técnicas de produção se configura como um ativo específico importante para a condução dos negócios, que permite que os CT sejam minimizados. No que se refere à tentativa de estabelecer um novo patamar de relacionamento entre as organizações que permita maior cooperação e reciprocidade, ficou claro que as variáveis as quais as empresas buscaram minimizar são exatamente o oportunismo e a racionalidade limitada dos empresários. Também o fato de a AGIVEST trabalhar com uma marca própria e incentivar uma maior especialização a partir da segmentação das ações ao longo da rede indica a tentativa de garantir para os consumidores um mix de produtos de maior qualidade. Os esforços são no sentido de reduzir os CT dos clientes da Rede, a partir de uma maior especialização, tecnificação e consolidação de uma boa imagem dos produtos com a marca AGIVEST. 
FÓRUM - SOCIOLOGIA ECONÔMICA

A PERSPECTIVA DOS CUSTOS DE TRANSAÇÃO NA FORMAÇÃO DE REDES DE COOPERAÇÃO

Alsones Balestrin - Alessandro Porporatti Arbage

Do ponto de vista da governança das ações organizacionais, identificou-se que ela é também dividida, mas, neste caso, em governança institucional e de gestão. A primeira é realizada pelo SEDAI, órgão do governo do Estado que organizou as ações para implantação e consolidação da Rede. Já, a governança em termos de gestão da Rede é estabelecida pela Assembléia dos Associados, que termina por definir os rumos operacionais e estratégicos da AGIVEST.

Quadro 2. Síntese das Principais Evidências Empíricas da Pesquisa

\begin{tabular}{|c|c|c|}
\hline \multirow{2}{*}{$\begin{array}{c}\text { Custos de Transação } \\
\text { e Governança }\end{array}$} & Tecnópole & AGIVEST \\
\cline { 2 - 3 } Racionalidade & Informações técnicas e de mercado & Informações técnicas, de mercado e de gestão \\
\hline Incerteza & Incerteza Secundária & - \\
\hline $\begin{array}{c}\text { Oportunismo } \\
\text { Especificidade de } \\
\text { ativo }\end{array}$ & - & Cooperação \\
\hline $\begin{array}{c}\text { Freqüência de } \\
\text { transações }\end{array}$ & Conhecimento & Conhecimento \\
& Marca & Marca \\
\hline $\begin{array}{c}\text { Forma de } \\
\text { Governança }\end{array}$ & - & Institucional (SEDAI) \\
\hline
\end{tabular}

O Quadro 2 apresenta uma síntese comparativa dos principais aspectos relacionados com os CT identificados na pesquisa, assim como as formas de governança que presidem as relações interorganizacionais nos arranjos estudados.

\section{CONSIDERAÇÕES FINAIS}

A presente pesquisa teve como principal finalidade contribuir para uma melhor compreensão da dinâmica de funcionamento de redes de cooperação, da perspectiva dos CT. Seu ponto de partida foi, sobretudo, a tese de Jarillo (1988), segundo a qual as relações de cooperação estabelecidas no contexto de uma rede possibilitam a redução dos $\mathrm{CT}$ entre outros aspectos significativos. 
FÓRUM - SOCIOLOGIA ECONÔMICA

A PERSPECTIVA DOS CUSTOS DE TRANSAÇÃO NA FORMAÇÃO DE REDES DE COOPERAÇÃO

À luz da ECT pôde-se perceber que as categorias analíticas ma is relevantes para compor redes de cooperação foram a racionalidade e a especificidade de ativos. Também foram citados, mas em menor grau de importância, a incerteza e o oportunismo.

Em termos de racionalidade, o que se observou foi a tentativa de ampliação das relações com outros atores econômicos com o objetivo de melhorar o processo de tomada de decisão. Em termos de especificidade de ativos, o que se identificou foi a tentativa de união de esforços visando ao desenvolvimento de conhecimentos para atividades conduzidas em conjunto, assim como a consolidação de uma marca própria.

As evidências demonstraram que, mesmo em contextos institucionais diferentes, as redes estabelecidas têm contribuído para uma maior dinâmica de interação entre as empresas participantes. A estrutura física criada facilita o compartilhamento de conhecimentos com implicações significativas nos processos de produção, aprendizado organizacional, gestão e inovação tecnológica. Adicionalmente, foi importante observar a atuação do poder público na governança institucional, que, aliada à governança mais operativa, definida de forma particular a cada situação, levou a um cenário de complementariedade no processo de coordenação.

Finalizando e utilizando uma tese sustentada pelo sociólogo Émile Durkheim, segundo a qual todo fato social merece, ao menos, duas interpretações, ponderamos que a ECT é uma das lentes possíveis de ser utilizada para compreender a natureza das relações que se estabelecem entre as organizações. Do ponto de vista das Teorias Organizacionais, a Teoria Institucional e a Teoria da Contingência Estrutural podem também ser "boas lentes" para a continuidade das pesquisas. No que diz respeito às Teorias Econômicas, a Visão Baseada em Recursos e a Teoria da Agência podem contribuir para uma interpretação mais ampla do processo de formação e gestão de redes de cooperação. No mais, consideramos que o fenômeno redes de cooperação deve merecer atenção de múltiplas lentes teóricas, visando a uma melhor compreensão e, sobretudo, à ind icação dos caminhos a serem seguidos objetivando a construção de arranjos interorganizacionais mais eficientes e eficazes.

\section{REFERÊNCIAS}

ARBAGE, A. P. Custos de transação e seu impacto na formação e gestão da cadeia de suprimentos: Estudo de caso em estruturas de governança híbridas do sistema agroalimentar no Rio Grande do Sul Tese de Doutorado, Programa de Pós-Graduação em Administração, PPGA/UFRGS, 267 p., 2004. 
FÓRUM - SOCIOLOGIA ECONÔMICA

A PERSPECTIVA DOS CUSTOS DE TRANSAÇÃO NA FORMAÇÃO DE REDES DE COOPERAÇÃO

Alsones Balestrin - Alessandro Porporatti Arbage

ARROW, K. The organization of economic activity: issues pertinent to the choice of market versus nonmarket allocation. In: THE ANALYSIS AND EVALUATION OF PUBLIC EXPENDITURE: THE PPB SYSTEM. Comité Económico Conjunto de los Estados Unidos, 91․ Congresso, $1^{\text {a }}$ sesión, Washington, D. C.: U.S. Government Printing Office, 1969.

BALESTRIN, A.; VARGAS, L.; FAYARD, P. O efeito rede nos pólos de inovação: um estudo comparativo. Revista de Administração (RAUSP), v. 40, n. 2, p. 159-171, 2005.

BARZEL, Y. Measurement cost and the organization of markets. Journal of Law and Economics, v. 25, n. 1, p. 27-48, 1982.

BURT, R. S. Structural holes. Cambridge: Harvard University Press, 1992.

CAGLIO, A. Networks and information technology: Competing through extranets. In: III CEMS COMMUNITY OF EUROPEAN MANAGEMENT SCHOOLS. Louvain-la-Neuve, 7/9 maio/ 1998.

CASTELLS, M. A era da informação: economia, sociedade e cultura. São Paulo: Paz e Terra, 1999.

CHANDLER, A. D. The Visible Hand. Cambridge: Harvard University Press, 1977.

COASE, R.H. The Nature of the Firm. Econômica, v. 4, n. 16, p. 386-405, 1937.

COMMONS, J. R. Legal Foundations of Capitalism. Macmillan: New York, 1924.

COMMONS, J. R. Institutional Economics. American Economic Review, v. 21, n. 4, p. 648-657, 1931.

DIMAGGIO, P.; POWELL, W. The iron cage revisited: institutional isomorphism and collective rationality in organizational fields. American Sociological Review, v. 48, n. 2, p. 147-160, 1983.

EGGERTSSON, T. Economic Behavior and Institutions. Cambridge, Survey of Economic Literature. Cambridge Univ. Press, 1990. 
FÓRUM - SOCIOLOGIA ECONÔMICA

A PERSPECTIVA DOS CUSTOS DE TRANSAÇÃO NA FORMAÇÃO DE REDES DE COOPERAÇÃO

Alsones Balestrin - Alessandro Porporatti Arbage

ECCLES, R. J. The quasi firm in the construction industry. Journal of Economic Behavior and Organizations, v. 2, n. 3, p. 335-357, 1981.

FAYARD, P. O jogo da interação: informação e comunicação em estratégia. Caxias do Sul: EDUCS, 2000.

GRABHER, G. The Embedded Firm: The Socio-Economics of Industrial Networks. London: Routledge, 1993.

HUMAN, S. E.; PROVAN, K.G. An emergent theory of structure and outcomes in small-firm strategic manufacturing network. Academy of Management Journal, v. 40, n. 2, p. 368-403, 1997.

JARILLO, J. C. On strategic networks. Strategic Management Journal, v. 9, n. 1, p. 31-41, 1988.

JOSKOW, P. L. The new institutional economics: alternative approaches. Journal of Institutional and Theoretical Economics, v. 151, n. 1, p. 248-259, 1995.

MARCON, C.; MOINET, N. La stratégie-réseau. Paris: Éditions Zéro Heure, 2000.

NOHRIA, N.; ECCLES, R. Networks and organizations: structure, form and action. Boston: Harvard Business School Press, 1992.

OLIVER, A.L.; EBERS, M. Networking network studies: an amlysis of conceptual configurations in the study of inter-organizational relationships. Organization Studies, v. 19, n. 4, p. 549-583, 1998.

PERROW, C. Small-Firm Networks. In: NOHRIA, N.; ECCLES, R. Networks and organizations: structure, form and action. Boston: Harvard Business School Press, 1992.

PERUCCI, R.; POTTER, H. R. Networks of power. Berlin: de Gruyter, 1989. 
FÓRUM - SOCIOLOGIA ECONÔMICA

A PERSPECTIVA DOS CUSTOS DE TRANSAÇÃO NA FORMAÇÃO DE REDES DE COOPERAÇÃo

Alsones Balestrin - Alessandro Porporatti Arbage

PFEFFER, J.; SALANCIK, G. R. The external control of organizations: a resource dependence perspective. New York: Harper and Row, 1978.

POWELL, W.W.; KOPUT, K.W.; SMITH-DOERR, L. Inter-organizational collaboration and the locus of innovation: networks of learning in biotechnology. Administrative Science Quarterly, v. 41, n. 1, p. 116-145, 1996.

REZENDE, D. C. Integração entre a ECT e o enfoque evolucionista: um estudo de caso no agribusiness do leite. In: ENCONTRO ANUAL DA ASSOCIAÇÃO NACIONAL DE PROGRAMAS DE PÓS-GRADUAÇÃO EM ADMINISTRAÇÃO, 22., 1999. Foz do Iguaçu. Anais. Foz do Iguaçu: ANPAD, 1999.

SABEL, C. Moebius-strip organizations and open labor markets: some consequences of the reintegration of conception and execution in a volatile economy. In: COLEMAN, J.; BOURDIEU, P. (Eds). Social Theory for a Changing Society. Boulder: Westview Press, 1991.

SALANCIK, G. R. A good network theory of organization. Administrative Science Quarterly, v. 40, n. 2, p. 345-349, 1995.

SAXENIAN, A. Regional Advantage: Culture and Competition in Silicon Valley and Route 128. Cambridge: Harvard University Press, 1994.

TEECE, D.J. Economies of scope and the scope of enterprise. Journal of Economic Behavior and Organization, v. 1, n. 3, p. 223-247, 1980.

TURATI, C. Economia ed organizzazione delle joint-ventures. Milan: EGEA, 1990.

WACHEUX, F. Méthodes Qualitatives et Recherche en Gestion. Paris: Economica, 1996.

WILliAMSON, O. E. Markets and Hierarchies: Analysis and Antitrust Implications. New York: Free Press, 1975. 
FÓRUM - SOCIOLOGIA ECONÔMICA

A PERSPECTIVA DOS CUSTOS DE TRANSAÇÃO NA FORMAÇÃO DE REDES DE COOPERAÇÃO

Alsones Balestrin - Alessandro Porporatti Arbage

WILLIAMSON, O. E. Transaction-cost economics: the governance of contractual relations. Journal of Law and Economics, v. 22, n. 2, p. 223-261, 1979.

WILliamsON, O. E. Las Instituciones Económicas del Capitalismo. México: Fondo de Cultura Económica, 1989.

YIN, R. K. Case Study Research: Design and Methods. 2. ed. California: Sage Publications, 1994.

\title{
Artigo recebido em 28.09.2005. Aprova do em 24.01.2007.
}

\author{
Alsones Balestrin \\ Doutor e Mestre em Administração pela Universidade Federal do Rio Grande do Sul. Professor e \\ Pesquisador do Programa de Pós-Graduação em Administração da Universidade do Vale do Rio dos \\ Sinos (UNISINOS) e Professor Associado do Instituto de Administração de Empresas da Universidade \\ de Poitiers (França). \\ Interesses de pesquisa nas áreas de gestão de redes interorganizacionais e gestão do conhecimento e da \\ inovação. \\ E-mail: abalestrin@unisinos.br. \\ Endereço:UNISINOS, Avenida Unisinos, 950, São Leopoldo - RS, 93022-000.
}

\begin{abstract}
Alessandro Porporatti Arbage
Doutor em Administração pela Universidade Federal do Rio Grande do Sul. Mestre em Econômia Rural pela UFRGS. Professor de Economia Rural nos Cursos Ciências Agrárias da UFSM. Professor de Análise de Cadeias Produtivas para o Curso de Pós-graduação em Extensão Rural da UFSM.

Interesses de pesquisa nas áreas de gerenciamento de cadeias de suprimentos de organizações agroindustriais, estratégias organizacionais e interorganizacionais, redes de cooperação, coordenação de cadeias produtivas agroindustriais e análise de mercados agrícolas.

E-mail: aparbage@smail.ufsm.br.

Endereço: Universidade Federal de Santa Maria - Fx de Camobi, km 9, Santa Maria - RS, 97119-900.
\end{abstract}

